Correo: innova@uide.edu.ec

\title{
Falla de estado y pérdida de bienestar para la sociedad
}

\section{Failure of state and loss of welfare for society}

Jaime Leopoldo Cabezas Maldonado

Universidad Internacional del Ecuador, Ecuador

Autor para correspondencia: jacabezasma@uide.edu.ec

Fecha de recepción: 27 de Junio de 2017 - Fecha de aceptación: 15 de Septiembre de 2017

\section{Resumen}

Se ha descrito en los textos que tratan la economía del sector público como parte de la microeconomía, sobre lo costosa que es para la sociedad la falla de mercado: a saber externalidades negativas como la contaminación, provisión insuficiente de bienes públicos y el monopolio que abusa de su poder de mercado, entre otras; pero poco se menciona sobre la falla del Estado, en que consiste, cual es el comportamiento del actor económico gobierno y como su accionar interfiere en el desarrollo de mercados sanos y competitivos, generando una inmensa destrucción de valor y riqueza, que se aprecia en pérdida de bienestar o pesos muertos en la sociedad. A continuación se enfatiza sobre la corrupción y las políticas de tipo populista como las fallas de Estado o Gobierno más graves incluyendo el enorme tamaño medido en término del ingreso nacional que está alcanzando el Estado del Bienestar.

Palabras clave: estado del bienestar; falla de estado; falacia del nirvana; corrupción; populismo

\begin{abstract}
It has been described in texts dealing with the economics of the public sector as part of the microeconomics, about how expensive market failure is for the society: negative externalities as pollution, inadequate provision of public goods and the monopoly and abuse of market power, among others; but little is mentioned about the failure of the State, that is, which is the behavior of the Government economic actor and as its powering interferes with the development of healthy and competitive markets, generating a huge destruction of value and wealth, which can be seen in loss of welfare or dead weights in society. Then we emphasize on the favor and the populist policies as more severe failure including the huge size measured in terms of National Income State that the welfare state is reaching.
\end{abstract}

Key words: state of well-being; state failure; nirvana fallacy; corruption; populism 


\section{Introducción}

¿Hasta dónde ha llegado la teoría sobre la falla de Estado? ¿Es adecuadamente estudiada esta falla? O la corriente principal - mainstream - de la ciencia económica neoclásica avasallada por la política, se ha convertido en sirviente del Estado del bienestar, representado en las corrientes antiliberales de la tercera vía, la economía mixta, la socialdemocracia y el socialismo de todas sus vertientes, tendencia que ha ido tomando cada vez más cuerpo y presencia en el mundo, siendo esto mismo la causa principal para la falla de Estado o Gobierno.

Este análisis verifica si la teoría de la falla de Estado se encuentra en un estado inteligible, si forma parte con el suficiente énfasis dentro de la economía neoclásica. Si es parte de una visión del mundo de la economía y de la filosofía política, verificar si la difusión de esta teoría es capaz de explicar positivamente este hecho económico y si normativamente sirve como una guía para la acción; por ejemplo, la propuesta de políticas públicas que limiten al Gobierno, o que su daño se minimice con la ejecución de algunas buenas prácticas o políticas públicas sanas, de haberlas. El objetivo de este trabajo es exponer que la falla de Estado no se ha enfatizado suficientemente, lo cual ha permitido que la ciencia económica oficial, presente a la falla de mercado como justificativo para una mayor intervención estatal cada vez, lo que se expresa en la regulación sobre el abuso del poder de mercado ${ }^{1}$.

Se ha definido a la poca información, al control limitado de las respuestas tanto del mercado como de la burocracia y a las limitaciones impuestas por el proceso político, como las únicas fallas del Estado. Viéndolo así, es muy poco lo que se ha podido hacer y concebir sobre los excesos del Estado, el cual ha seguido creciendo insosteniblemente. (Stiglitz, 2000). Para justificar el quehacer del Estado del Bienestar, se han incrementado los presupuestos de casi todos los gobiernos a nivel mundial de manera exorbitante, creciendo durante el siglo XX en ratios del PIB desde un 5\% a alrededor del 50\% en tiempos de paz, por tanto cada individuo en la sociedad se ha visto obligado a contribuir con su renta de manera creciente para sostener una inmensa y costosa burocracia, autocumpliéndose la profecía de Hayek dada en los años cuarenta del siglo pasado de que el mundo caminaba a pasos acelerados por el "camino de servidumbre". La escuela austríaca de economía es la que mejor ha expuesto la gravedad de toda forma de estatismo, la cual le estaría llevando al mundo a nuevas formas de autoritarismo y despotismo. (Hayek, 2007) (Hoppe, 2015). Se afirma que las fallas de Estado son cuantitativamente y cualitativamente más profundas y dañinas que las fallas del mercado, haciendo énfasis en lo siguiente:

En la primera parte se desarrolla el tema de la corrupción de los funcionarios públicos como aquellos fenómenos que se analizan con el modelo de agencia y captura, (Stigler, 1978) y luego se muestran los estudios de tipo normativo para la implementación de estrategias anticorrupción. (Klitgaard, 2000). Se describe la teoría de la elección pública la cual permite ver a "la política sin romanticismo", en la que los teóricos de esta escuela, demuestran que la clase política trabaja para sus propios intereses, por lo cual el bien común solo es una coartada. (Buchanan, 2001). El estudio muestra que se forma una casta amalgamada de políticos y falsos

\footnotetext{
${ }^{1}$ Regulación sobre monopolios y oligopolios, externalidades negativas como la contaminación o sobre el que y el cómo de la provisión de bienes públicos, sistema tributario y efectos de los impuestos en la demanda y comercio. (Mankiw, 2012) (Samuelson \& Nordhaus, 2010)
} 
empresarios -compadres- amparados en este Estado intervencionista, - en la versión marxista sería el capitalismo monopolista de Estado- es decir, pseudo empresarios que capturan rentas basados en el proteccionismo promovido por el Estado. Estos grupos de interés usando la retórica, se toman el poder y tratan de maximizar en todo momento su capital político que se transforma en un mecanismo de dominación, ya que tienen el monopolio de la fuerza, tanto para extraer rentas vía impuestos, cuanto para reprimir por la vía de la violencia de la fuerza pública y todo el aparataje institucional creado, a todos aquellos que no se adecúen a esta construcción, todo a nombre de un mal entendido bien común. El más importante bien común es la libertad individual, el derecho a la vida y a la propiedad personal.

En la segunda parte se desarrolla la macroeconomía del populismo (Dornbush, Rudiger y Edwards, Sebastian, 1991) con su base teórica y nuevos casos. Describe como esta forma de armar la política económica redistributiva, destruye el sistema de precios, hace cambios en la estructura de la propiedad privada, lo que desincentiva la inversión productiva de tipo competitivo y favorece la creación de una clase social parasitaria que vive del Estado benefactor. Se demuestra que la implementación de estas políticas una vez que los fondos se agotan, la economía cae en picada con un costo social alto que recae precisamente con más fuerza sobre las personas más débiles de la sociedad. En efecto un país en el que se ha aplicado un plan macroeconómico populista, termina en una profunda crisis por la imposibilidad del manejo del déficit fiscal. Finalmente cabe aplicar programas de ajuste y choque, para revertir este tipo de efectos siendo el más obvio, el recorte del gasto público y por ende buena parte de los subsidios, bajo un ambiente de recesión, desempleo y crisis cuyo costo social es inmenso.

En la parte final con base a la "falacia del nirvana" (Cabieses, 2012), se descubre como la creencia de que el Estado providencial del bienestar, creación intelectual profundizada a partir de la posguerra con base a los postulados de la economía keynesiana, promueve la economía mixta usando el constructivismo o ingeniería social en donde una gran cantidad de bienes y servicios se han vuelto públicos por decreto, dando como resultado un nivel de falla de Estado, destructor de riqueza y bienestar para la sociedad por los efectos microeconómicos.

\section{Corrupción}

La corrupción ha sido definida como el abuso del poder encomendado para beneficio personal. Todos los días se escucha sobre el enriquecimiento ilícito de burócratas, entendiéndose como tales desde jefes de gobierno hasta servidores de menor jerarquía, quienes no tienen como justificar el incremento de su patrimonio, debido a la falta de legitimidad de sus ingresos. Los sobornos desde el corruptor hacia el corrupto, se dan pues este tiene discrecionalidad en procesos de compras públicas o cualquier manejo de recursos públicos, se descubran o no ante la opinión pública, debido a la falta de transparencia y controles externos. Esta situación se sustenta en un hecho cierto; quienes acceden al Estado manifiestan su propio interés para maximizar sus beneficios, lo cual no es una situación excepcional sino más bien una constante.

Si bien hay muchas teorías, (Gómez-Alvarez Díaz \& Gónzalez, 1998) y otros en el que al menos se citan a más de noventa autores que han dado muchas interpretaciones y propuesto estrategias anticorrupción, más bien se usa la modelización -jefe-agente-cliente- en donde el jefe es la ley, la autoridad que ordena un procedimiento racional y correcto, es decir el sistema que 
delega a un agente o agentes, típicamente un político o un burócrata, un determinado procedimiento pegado a una normativa racional y ética, agente que tienen discrecionalidad para decidir a su antojo en la medida de la debilidad del jefe y finalmente, el cliente, es decir la sociedad formada por los individuos que serían las víctimas del comportamiento corrupto, unas veces como aquellas personas que deben pagar el sobreprecio y otras veces como quienes se someten a una carga administrativa y tributaria costosa para hacerse acreedores a un determinado contrato o servicio público. Se ha sentenciado que la corrupción es una enfermedad pandémica y contagiosa difícil de combatir, observada en todos los países pero con incidencia nociva más fuerte en algunos de ellos. (Klitgaard, 2000)

Una afirmación intuitiva pero no divorciada de la realidad sería ¿a cuánto del ingreso nacional (PIB) equivale este comportamiento? Si la carga presupuestaria mundial de las economías para mantener al Estado es de un 30\% y la corrupción representa $10 \%$, podría decirse que este costo es de al alrededor de un $3 \%$ de la renta nacional.

Según el Índice de Percepción de la Corrupción 2014 (Transparencia Internacional, 2014) se manifestó que cuando los altos funcionarios abusan de su poder para usar fondos públicos en beneficio propio, el crecimiento económico se ve minado y los esfuerzos por frenar la corrupción quedan frustrados. El informe indicó que más de dos tercios de los 175 países incluidos en este índice obtuvieron una puntuación inferior a 50, en una escala de 0 equivalente a percepción de altos niveles de corrupción, y de 100 como la percepción de bajos niveles de corrupción. Una mala puntuación evidencia un contexto donde prevalece el soborno, los actos de corrupción quedan impunes y las instituciones públicas no dan respuesta a las necesidades de los ciudadanos.

Una ecuación que se usó en las aulas por algún tiempo definía a la corrupción como proporcional a la discrecionalidad de los agentes que toman decisiones a nombre del Estado más el grado de monopolio o poder de mercado de determinadas industrias que reciben las prebendas, e inversamente proporcional a los niveles de transparencia, información y control. Sin embargo por cultura, los ciudadanos se han acostumbrado a vivir con este mal que es consustancial a la existencia del Estado.

El nivel de corrupción de una sociedad, es directamente proporcional a su grado de estatización; cuanto mayor sea el nivel de intervención en la economía, mayores serán las modalidades de corrupción que afloren. En un sistema intervencionista, al margen de la utilidad del burócrata o de su desprendido servicio a la comunidad, existe el permanente riesgo de que aquellos que tienen la administración del gobierno se retribuyan por encima de lo suficiente, convirtiendo su cargo en un instrumento de enriquecimiento personal... la presencia de la corrupción en los sistemas democráticos, tomará a éstos en un mecanismo de compra de votos para fabricar una clientela fiel al poder. (Quiróz, 1998)

Nótese la relación existente entre el tamaño del Estado y su nivel de intervención, entiéndase como limitación de la libertad individual, de asociación, de pensamiento y de opinión, es decir un sistema que imposibilita la transparencia debido al miedo impuesto y sigilo imperante. Un caso especial que es parte de la teoría de la corrupción conocida como búsqueda de rentas, situación en la que el Estado desarrolla programas de asignación de recursos mediante 
transferencias a quienes se acogen a un diseño institucional en el que el sistema de precios se lo define de una manera discriminatoria y monopolística, muchas veces de manera contraria de lo que sería el mercado y un precio competitivo. Así en forma artificial se va forjando un mecanismo que posibilita maximizar rentas y beneficios monopolísticos financiados por el Estado, es decir por los contribuyentes. El que captura estos recursos muchas veces como resultado de un lobby es el buscador de rentas, quien supervive en la medida en que el programa de gobierno funciona. Los mercados administrados por el Estado dan lugar a este fenómeno. (Boehm, 2005)

Esta situación se liga a la protección de la industria naciente, es decir a todas las industrias subsidiadas como parte de los modelos de sustitución de importaciones con sesgos anti exportadores, a los programas de capacitación y formación subsidiados por los contribuyentes, a los grupos de beneficiarios de bonos o seguros de desempleo, de manera universal e indefinida. A todos estos grupos de beneficiarios que han ido creciendo insosteniblemente en el tiempo, se los utiliza y fideliza al gobierno paternalista que hace uso de estas prácticas, a cambio de votos. Incluso este modelo es más sofisticado en países como España, Grecia, Italia y Portugal, que ha fomentado en los ciudadanos una necesidad de amplificar el Estado del Bienestar, encargado de proteger a los individuos de la cuna a la tumba, bajo un incentivo perverso al impedir que cada ser humano se responsabilice de su búsqueda personal, libre y soberana de su propio progreso y felicidad. Al respecto Sen ve en la libertad individual el mayor incentivo para que cada ser humano se responsabilice de sí mismo y así se evite convertir a ciertos seres humanos en parásitos sociales que viven del resto. (Edo, 2012) Esto profecía ya fue descrita en el siglo XIX cuando se afirmó que el Estado es la gran ficción a través de la cual todo el mundo se esfuerza en vivir a expensas de todo el mundo. (Bastiat, 2004)

Una de las consecuencias de este Estado benefactor es que se ha creado una subclase de beneficiarios de estos programas del bienestar, quienes desconocen lo que es el trabajo. Se reduce el potencial productivo de la economía nacional, mientras al mismo tiempo los programas del Estado benefactor no logran satisfacer eficazmente ninguna de los objetivos que dieron lugar a su implementación. Luego de presentar este diagnóstico, resulta mucho más difícil individualizar alguna reforma eficaz y no es fácil desmantelar el Estado benefactor. (Buchanan J. , 1988)

\section{Populismo}

La Macroeconomía del populismo es un diseño de políticas públicas macroeconómicas, aunque impactan potentemente en la microeconomía. Se demostró que aquellos países en donde se aplicaron estas estrategias anti mercado agresivas, partían de precondiciones tales como que esas economías tenían demasiados fondos ociosos de ahorro y capital físico, mientras supuestamente el pueblo sufría de pobreza. Los indicadores de tipo macroeconómico estaban en relativo orden, debido a políticas económicas ortodoxas previamente implementadas con el fin de mantener estables el sector fiscal y el externo; en cambio, en contraposición el crecimiento económico era lento y existía preocupación por la concentración del ingreso.

La prescripción de políticas populistas con diferentes matices fueron: usar y consumir los fondos ahorrados para apalancar el crecimiento por la vía de un creciente gasto público, 
incremento de subsidios universales y regresivos como, el de los combustibles y otros focalizados hacia los más pobres tales como seguros de desempleo u otro tipo de ayudas y crecimiento del empleo en el sector público para recrear esta nueva institucionalidad intervencionista.

En la mayoría de economías, como se contaba con política monetaria propia, el banco central usaba activas políticas crediticias y creación de dinero primario, para financiar crecientes déficits fiscales y planes de crédito segmentado y subsidiado para vivienda e industria. La política industrial consistía en promover la primera etapa de la Industrialización Sustitutiva de Importaciones, con una orientación de crecimiento hacia adentro y con un sesgo anti exportador debido a que se aplicaban tasas de protección efectiva que en algunos casos hasta superaban el $100 \%$. Este proteccionismo ha sido una constante en los procesos de industrialización en casi todas las economías latinoamericanas donde se aplicaron desde los años 50 y que penosamente los industrializadores tardíos y reincidentes, los han vuelto a aplicar en lo que va de este siglo. (Urriola, 1988) (Salcedo, 2009)

Debido a las presiones inflacionarias se profundizaban los controles de precios fijados políticamente, con las consecuentes distorsiones, como escasez generalizada que se iba agravando o exceso de producción que al pasar del tiempo terminaban quebrando la economía. Dada la crisis de no sostenibilidad de este modelo, profundizaba la aplicación de subsidios por montos que alcanzaron varios puntos del PIB. Una característica de estos programas incluía importantes afectaciones a la propiedad: expropiaciones, confiscaciones y nacionalizaciones; por tanto se pusieron a la orden del día, reformas agrarias, nacionalización de bancos y de industrias de gran envergadura como la petrolera y minera, bastaba con calificarlas de industrias estratégicas. La aplicación de estos planes en la práctica daba como resultado el paso por cuatro fases:

En una primera, los gobiernos confirmaban su pronóstico: alto crecimiento de la renta nacional, debido al consumo de los fondos de ahorro y eventualmente al alto precio de materias primas de exportación producto de todo tipo de minería y agricultura; mejora del salario real, tasas de empleo elevado; los controles sostenían la inflación, alta popularidad de los gobiernos en esta fase, en la que si llamaban a elecciones, el triunfo electoral era una constante una y otra vez. Una buena parte de la sociedad estaba contenta con un gobierno que había recurrido a un permanente estado de propaganda y movilización al despertar la pasión que provoca en los individuos la lucha de clases, simplificada en pobres contra ricos supuestos causantes de todos los males de la sociedad.

Pero este orden de eventos no era sostenible en el tiempo, pues había sectores de la sociedad golpeados por la agresividad de una serie de políticas públicas, como las expropiaciones y la violencia de estos regímenes. A pesar del crecimiento económico, ha existido una destrucción de patrimonio y los fondos de ahorro están casi exhaustos. La existencia de los déficits gemelos, fiscal y externo, en donde el primero era el causante del segundo, estaba empezando a hacer aflorar la crisis, pasando así a una segunda fase en que la economía presentaba estrangulamientos: comenzaban a faltar las divisas, la realineación de precios se volvía una necesidad debido a los retrasos en los ajustes por los crecientes costos, la devaluación, el control de cambios o más protección se volvían indispensables y eran recurrentes para sostener 
el modelo, lo que terminaba empeorando los precios relativos de los bienes comercializables. Los inventarios estaban al mínimo y empezaba la escasez.

En una tercera fase, dados los cambios en la propiedad privada, los controles de precios, el alto desempleo, la escasez generalizada, provocaba una fuga de capitales masiva y la desmonetización de la economía. Los salarios reales caían, los déficits aumentaban. Como el déficit fiscal fácilmente podía alcanzar dos dígitos del PIB, se caminaba a pasos agigantados a la hiperinflación pues la única forma de sostener al gobierno era con emisiones cuantiosas.

En la cuarta fase con el gobierno populista o sin él, se requería la aplicación de un modelo ortodoxo, conservador y prudente, que haga exactamente todo lo contrario. El país ha quedado peor que antes de la aplicación del programa populista y los individuos, especialmente los más débiles eran los grandes perdedores. (Paredes, 1996)

Como lo manifestó este autor, estos programas tenían su raíz en una visión de corte populista orientada por la buena fe y el deseo de ayudar a los desposeídos. Pero la puesta en marcha de sus políticas chocaba con la realidad. Creer que el déficit fiscal no importa, o que no pasa nada si se consumen las reservas de ahorro, o crear dinero sin respaldo, son políticas que acababan desequilibrando la economía. Para Ilustrar, veamos indicadores selectos de Chile durante el gobierno de la Unidad Popular:

Tabla 1

Indicadores macroeconómicos de Chile

\begin{tabular}{lccr}
\hline Chile: picos entre 1970 y 1973 & $\begin{array}{c}\text { fase 1 } \\
\text { Éxito }\end{array}$ & $\begin{array}{c}\text { fase } 2 \\
\text { Ambigüedad }\end{array}$ & $\begin{array}{c}\text { fase 3 } \\
\text { Descalabro }\end{array}$ \\
\hline Crecimiento PIB\% & 9 & $-1,2$ & $-5,6$ \\
\hline Inflación \% & 35 & 217 & 606 \\
\hline Salario Real base 100 & 98 & 115 & 70 \\
\hline Déficit fiscal como \% del PIB & 2,7 & 10,7 & 24 \\
\hline Reservas en millones de US\$ & 320 & 129 & 36 \\
\hline Balanza Comercial en millones de US\$ & 246 & 73 & -161 \\
\hline $\begin{array}{l}\text { Tasa de crecimiento del dinero, fuente de la } \\
\text { inflación }\end{array}$ & 53 & 101 & 264 \\
\hline $\begin{array}{l}\text { Nota: Tomado de "La macroeconomía del populismo” por Noel Ramírez.- INCAE, Quito, 1988.- Adaptación de } \\
\text { este trabajo por parte del autor }\end{array}$ & &
\end{tabular}

Los expertos consideraron sí podrían haber variantes en los países que se aplicaron programas populistas tales como los gobiernos peronistas de Argentina de la era Kirchner o Venezuela con Chávez y su sucesor, insinuando que la duración de la fase uno podría ser extensa si se contaba con los recursos. Con ese enfoque, este sería el caso de Venezuela entre 2000 y 2015, (aun cuando hay opacidad en la información), país que podría estar entrando ya en la fase tres, pero que hasta el año 2014, vivió una larga fase 1 debido al billón de dólares (trillón en inglés) recibidos como parte de la renta petrolera de estos quince años:

Tabla 2

Indicadores macroeconómicos de Venezuela 


\begin{tabular}{|c|c|c|c|}
\hline Venezuela: picos entre 2000 y 2016 & $\begin{array}{l}\text { fase } 1 \\
\text { Éxito }\end{array}$ & fase 2 Ambigüedad & fase $3 * \quad$ Descalabro \\
\hline Crecimiento PIB\% & 4,9 & -4 & -18 \\
\hline Inflación \% & 14 & 63 & 720 \\
\hline Salario Real base 100 & 600 & 100 & 90 \\
\hline Déficit fiscal como \% del PIB & -2 & -4 & -10 \\
\hline Reservas en millones US\$ & 43,000 & 23,000 & 12,168 \\
\hline Saldo en Cta Corriente \% PIB & 17,2 & 0,7 & -4 \\
\hline Tasa de crecimiento del dinero & 16 & 37 & 71 \\
\hline
\end{tabular}

Según ciertos políticos venezolanos lo que podría ser el resultado de una supuesta guerra económica, no sería sino la crónica de resultados que se los veía venir. Desgraciadamente en América Latina y algunos países europeos, el populismo con diversos matices, es una constante.

\section{A manera de conclusión la Falacia del Nirvana}

Desde la propia escuela marxista que es la parte vergonzante del maistream, se desmitificó el Estado del cual siempre se afirmó que es un mecanismo de opresión basado en el cobro de impuestos y el monopolio de la violencia institucional. El tema fue retomado por Weber, sin embargo, una idea generalmente aceptada es que se requiere del Estado para regular la sociedad que de otra manera caería supuestamente en el caos y anarquía. Esta simplificación aceptada generalmente por la mayoría de quienes profesan las ciencias sociales, ha servido para justificar el accionar del intervencionismo del moderno Estado Benefactor.

La visión expuesta estudia los "fracasos del gobierno" como una respuesta ante quienes sostienen que el gobierno debe intervenir ante los denominados fracasos del mercado; así se postula que la evidencia científica señala al gobierno -y no al mercado- como el ente que debe ser reducido para lograr el bienestar de la sociedad. Esta teoría coincide con la necesidad de un Estado limitado con el objeto de que esta institución al fin y al cabo regentada por seres humanos imperfectos, no se extralimite en las facultades sobre los individuos. Esta visión coincide con la de los próceres de los Estados Unidos de América, quienes tenían un temor muy grande ante el aparecimiento de una nueva fuerza opresora y despótica basada en el Gobierno. Vale decir que los padres fundadores fueron personas queridas y respetadas que vivieron una larga ancianidad, a diferencia de los libertadores latinoamericanos que fueron traicionados y abandonados; penosamente estos últimos no tenían claro que la libertad que se perseguía era la libertad individual y no solo la consecución del cambio de un gobierno colonial por uno criollo, en donde la libertad individual era secundaria y el despotismo de quienes hacen el Estado se ha mantenido hasta la presente. Esto ya fue visualizado por Alberdi, filósofo y político argentino del siglo XIX cuando demostró en un ensayo que la omnipotencia del Estado es la negación de la libertad individual. (Alberdi, 2003)

Citando a Cabieses, presenta la falacia del nirvana - término acuñado por el economista de la Escuela de Chicago Harold Demsetz quien introdujo el concepto en un famoso artículo que escribió en 1969, titulado Información y Eficiencia - como aquel error que consiste en comparar 
una situación real, por ejemplo un problema social, contra una solución idealizada y por tanto irreal. Por ejemplo quien puede resolver el problema del abuso del monopolio es el Gobierno, mediante ciertas políticas, siendo una de las más usadas la regulación per se en la que el propio Gobierno se convierte en el nuevo proveedor monopolista, es decir, se cambia un monopolio privado por uno público, pero esto no resuelve el problema, más aún lo empeora. Lo que debe hacerse es comparar alternativas reales:

El Estado está lejos de ser tal entidad infalible, por el contrario, su intervención suele generar más perjuicios que beneficios. Ejemplos hay miles, el sueldo mínimo genera desempleo, las protecciones a los consumidores generan alzas de precios, los impuestos desincentivan la generación de riqueza, las empresas estatales son altamente ineficientes, los programas de asistencia social suelen terminar siendo fuentes de corrupción, etc. Así, los argumentos en favor de la intervención del Estado, que se presentan como axiomas (premisas evidentes que no requieren una demostración alguna), están lejos de serlo." (Cabieses, La falacia del Nirvana, 2012)

\section{Bibliografía}

Alberdi, J. B. (2003). La omnipotencia del Estado es la negación de la libertad. Recuperado de http://www.elcato.org.La-omnipotencia-es-la-negación-de-la-libertad-individual, 1-19.

Bastiat, F. (2004). El Estado. Madrid: Unión Editorial.

Boehm, F. (2005). Corrupción y captura en la regulación de los servicios públicos. Economía Institucional, 245-260.

Buchanan, J. (1988). Consecuencias económicas del estado benefactor. Libertas, 1-9.

Buchanan, J. M. (2001). Ideas de Libertad. Mi peregrinaje intelectual (págs. 20-28). Guayaquil: Instituto Ecuatoriano de Economía Política.

Cabieses, G. (2012). La falacia del nirvana. Recuperado de http://www.elcato.org/la-falacia-delnirvana, 1-2.

Dornbush, Rudiger y Edwards, Sebastian. (1991). La Macroeconomía del Populismo en América Latina. En R. Noel, Economía y Populismo, Ilusión y Realidad en América Latina (págs. 39-89). Quito: INCAE.

Edo, M. (2012). Amartya Sen y el desarrollo como libertad. Revista de la Universidad Torcuato di Tella, 20-26.

Gómez-Alvarez Díaz, R., \& Gónzalez, L. (1998). Una revisión del análisis económico de la corrupción. Recuperado de https://dialnet.unirioja.es/descarga/articulo/3087254.pdf, 217.

Hayek, F. (2007). Camino de Servidumbre. Madrid: Alianza Editorial. 
Hoppe, H.-H. (2015). Socialismo y Capitalismo. Quito: Universidad San Francisco de Quito.

Klitgaard, R. (2000). Contra la corrupción. Finanzas \& Desarrollo, 2-5.

Mankiw, G. (2012). Principios de Economía. Mexico: Cengage Learning.

Paredes, P. L. (1996). Reflexiones al rededor de la economía del nuevo gobierno. Ekos, 15-18.

Quiróz, L. B. (1998). Estado, economía y corrupción. Dialnet, 255-262.

Salcedo, J. J. (2009). Industrialización por sustitución de importaciones. Recuperado de http//www.ecuadoreconómica, 1.

Samuelson, P., \& Nordhaus, W. (2010). Economia. Mexico: Mc Graw Hill.

Stigler, G. J. (1978). La aplicación óptima de las leyes. ICE, 8-15.

Stiglitz, J. (2000). La economía del sector público. Barcelona: Antoni Bosh - Universidad de Alcalá.

Transparencia Internacional. (2014). Indice de percepción de la corrupción. Recuperado de http://www.transparency.org/news/pressrelease/indice_de_percepcion_de_la_corrupcion _2014_, 5-15.

Urriola, R. (1988). Políticas de industrialización en America Latina. Quito: ILDIS. 